\title{
Tragende Netze und schwankende Böden: Neue Herausforderungen für Religionsgemeinschaften in der Migrationsgesellschaft
}

\author{
Martin Baumann / Klaus Hock / Wolfram Reiss
}

Immigration presents new challenges for European societies, Christian churches, and academic research. The paper argues that first of all a change in the terminology of describing the phenomenon of migration/immigration is needed. Secondly, it is of crucial importance to discern and appreciate the different educational services provided by religious immigrant communities, but likewise to consider and observe concurrent trends toward reinforcing different aspects of conservative or backward oriented power structures and liabilities. As for the Christian churches, they face new forms of internal plurality and multifaceted forms of spirituality due to major changes in their constituency, caused by a decrease in traditional membership and an influx of new members coming from immigrant communities. Academic research, being committed to critical impartiality, is facing major challenges in view of its task to analyse such dramatic transformations caused by immigration and religious changes in a sober, rational, and factoriented way in order to provide basic information for policy makers and civil society.

Martin Baumann has been professor of Religious Studies at the Faculty of Cultural and Social Sciences since 2001 and prorector for research at the University of Luzern since 2010. He teaches and investigates the fields of migration, religion and social integration; controversies and coexistence in religious pluralism; Diaspora Studies; Hindu and Buddhist traditions in the West. His most recent publication in co-authorship is Integrationspotenziale von Religion und Zivilgesellschaft, Baden-Baden 2016.

Klaus Hock is professor of History of Religion - Religion and Society at the Faculty of Theology at the University of Rostock; Moreover, he has been speaker (2010-2013), respectively deputy-speaker (2006-2013 and again since 2013), of the DFG-Graduate Training Group "Kulturkontakt und Wissenschaftsdiskurs" as well as founding member of the council of the DFG-Graduate Training Group "Deutungsmacht: Religion und Belief Systems in Deutungsmachtkonflikten" (since 2014). His research interests are: religion and transculturation; Christian-Muslim relations in Africa; religion in Africa and African religions, especially African Christianity.

Wolfram Reiss has been professor for the Studies of Religions at the Protestant Faculty of the University of Vienna since 2007. His main research interests are the religions in the Middle East; the impact of immigration on institutions in Europe; recent developments in policy, pedagogy and religions in Egypt and Syria. He edits the series "Application Oriented Studies of Religions" (AORW). Recent publications in this series (2016): 
Selbstbetrachtung hinter Gittern. Naikan im Strafvollzug in Deutschland und Österreich (AORW 6); Aufstieg und Fall der Muslimbruderschaft 2011-2013 (AORW 8).

\section{Einleitung}

Der Zusammenhang von Religion und Migration ist zu einem viel diskutierten Thema in der Politik, im Sozialbereich und in den Medien geworden. Nicht erst die Ankunft großer Kontingente von Flüchtlingen aus den Kriegs- und Krisengebieten Syrien, Irak und Afghanistan in den europäischen Ländern (2014/15) hat die Thematik von Migration in Kombination mit religiöser Zugehörigkeit in den Vordergrund gerückt. Schon seit den 1980er und 1990er-Jahren, als im Zuge neuer Zuwanderungen aus dem Nahen Osten und aus asiatischen Ländern MigrantInnen mit muslimischer, buddhistischer, hinduistischer oder anderer Religionszugehörigkeit nach Europa kamen und sich auf Dauer niederließen, bildete das Gespann von Religion und Migration ein gesellschaftspolitisches Reizthema. Es war die Zeit, als viele Politiker und Sozialwissenschaftler das soziale Verschwinden von Religion voraussahen, der nun jedoch im Zuge von verstärkter Immigration neues Leben eingehaucht zu werden schien. Die Problematisierung und zunehmende Stereotypisierung von Islam und MuslimInnen ab den 1990erJahren sowie verstärkt nach 2001, den Attentaten von Madrid und London sowie dem Karikaturenstreit (2006) hatte auf nationalkonservativer, anti-muslimischer Seite den Reflex ausgelöst, ein sogenanntes „christliches Abendland“ zu beschwören: Christlich-jüdische Tradition(en) in Gestalt der Postulierung einer ,jüdisch-christlichen Leitkultur“ sollen als Grenze und Bollwerk die vermeintliche Unterwanderung Europas durch Islam und muslimische ZuwanderInnen verhindern. ${ }^{1}$ Unverhofft für viele waren und sind die Themen Religion und Migration tages- und gesellschaftspolitische Aktualität geworden.

Der vorliegende Beitrag fragt nach der Identifikation gegenwärtiger und zukünftiger Herausforderungen, mit denen sich Wissenschaft, Politik, Zivilgesellschaft und christliche wie nicht-christliche Religionsgemeinschaften zu befassen haben. Welche neuen gesellschaftlichen Entwicklungen im Themenbereich Religion und Migration zeichnen sich ab, welche zukünftigen Entwicklungen stellen

1 Die Behauptung einer statischen ,jüdisch-christlichen Leitkultur“, in die sich andere einzufügen hätten und die Negierung islamischer und anderer religiöser Prägungen ist angesichts Jahrhunderte andauernder Migrations- und Transformationsprozesse in Europa höchst fragwürdig. Europa ist gerade nicht durch eine religiös-ethnische Homogenität, sondern durch Diversität, Konkurrenz, Konflikt, Koexistenz und kontinuierliche Transformationsprozesse charakterisiert, die sich in der Gegenwart nur wieder einmal verstärken und mit Sicherheit auch noch nicht abgeschlossen sind. Vgl. Reiss 2015, S. 143-149; Meyer 2013; Cohen 1995; Gotzmann et al. 2001; Elsas 2002; Kippenberg / Rüpke / von Stuckrad 2009. 
Herausforderungen für wissenschaftliche Forschung und sozialpolitische Praxis? Dieser Fragen nimmt sich der Beitrag anhand von drei separaten, jedoch eng miteinander verwobenen Zugängen an: Erstens, der Identifikation möglicher bzw. wahrscheinlicher gesellschaftspolitischer Entwicklungen in westeuropäischen Einwanderungsländern mit Blick auf Religion und Migration; zweitens, den Herausforderungen, denen sich christliche Kirchen und Religionsgemeinschaften angesichts künftiger gesellschaftspolitischer Entwicklungen stellen müssen; und drittens versucht der Beitrag auf der Grundlage der zwei zuvor dargestellten Themenblöcke darzulegen, welche neuen Forschungsfragen und Forschungsherausforderungen aus der Sicht der Religionswissenschaft und Religionsgeschichte identifiziert werden können. Angesichts der hohen gesellschaftspolitischen Aktualität von Religion und Migration ist die Religionsforschung in vielfacher Weise gefordert, sich aktiv der Thematik anzunehmen und in die Debatte einzubringen. Dabei wird es nicht nur darum gehen, auf der Grundlage wissenschaftlicher Differenzierung, Reflexion und Analyse ein möglichst sachadäquates Bild zu zeichnen, sondern zur politischen und zivilgesellschaftlichen Diskussion um Migration und Religion gleichermaßen kritische wie konstruktive Denkanstöße zu geben.

\section{Mögliche gesellschaftspolitische Entwicklungen der Thematik Religion - Migration}

Auf lediglich zwei Themen soll an dieser Stelle eingegangen werden. Für gesellschaftspolitisch wichtig halten wir einerseits die Frage adäquater Begrifflichkeiten und andererseits eine Analyse der medialen Verhandlung der Thematik.

Zur Frage adäquater Begrifflichkeiten: In Politik und Medien und vielfach in der Forschung ist von Migration bzw. von Migrantinnen und Migranten die Rede. MigrantInnen sind solche, die kommen, eine Weile bleiben und dann weiterziehen. Wir plädieren hingegen dafür, den Begriff der Migration mit einem Begriff zu ersetzen, der die Sachverhalte von Ankunft, Niederlassung und Einrichten auf Dauer adäquat benennt. Es ist der Begriff der Immigration. Viele Länder Kontinentaleuropas scheuten sich bis zu Beginn des 21. Jahrhunderts, sich als Einwanderungs- und Immigrationsländer zu bezeichnen. ${ }^{2}$ Doch genau dieses sind Länder wie Deutschland, die Schweiz, Österreich oder Schweden. Die einstigen

2 Vgl. Horst Seehofers noch 2010 vorgelegter Sieben-Punkte-Plan, nach dem Deutschland kein Einwanderungsland sei und Muslime selbst bei Erhalten der Staatsangehörigkeit nur eingeschränkte Rechte haben sollten (siehe Internetquellen: Focus Online). Kritisch dazu Thomas Assheuer und Ulrich Preuß, die auf die große Gefahr aufmerksam machen, wenn Rechtsgleichheit von der kulturellen und religiösen Zugehörigkeit abhängig gemacht wird. Vgl. Internetquellen: Assheuer 2010; Internetquellen: Preuß 2010 sowie die kritische Analyse des Sozialwissenschaftlers Nowak 2006. 
„GastarbeiterInnen“, Flüchtlinge, HeiratspartnerInnen und Hochqualifizierten sind im Land der Zuwanderung sesshaft, also zu ImmigrantInnen und „Einheimischen" geworden.

Die Perspektive der Wahrnehmung von Prozessen und Personen ändert sich, wenn sie als ImmigrantInnen und potenziell künftige Neubürger mit Stimmrecht angesehen werden. Als Neubürger bedürfen sie umfangreicher Maßnahmenpakete, finanzieller und organisatorischer Ressourcen, mit denen die sprachliche, kulturelle, soziale und religiöse Integration und die Identifikation mit der neuen Heimat von Anfang an gefördert werden. Nicht nur sie selbst, sondern auch der Staat ist für die Integration verantwortlich, da er selbst höchstes Interesse daran hat, seine neuen Bürger möglichst umfassend und schnell voll $\mathrm{zu}$ integrieren. Nicht noch einmal wie in den 60er-Jahren sollte versäumt werden, von Anfang an den neuen MitbürgerInnen ein umfassendes Angebot zum Erlernen der Sprache, zur beruflichen Integration, zur Vermittlung von Geschichte und Kultur und von gesellschaftlichen Grundwerten anzubieten. Solche Maßnahmenpakete können sicherlich eine raschere Anerkennung und Integration bewirken, jedoch auch Ängste, Neid und Abwehrreaktionen in bestimmten Bevölkerungsteilen hervorrufen oder verstärken.

Überdies gilt es jedoch, noch einen Schritt weiterzugehen. Denn ein Teil der „ImmigrantInnen“, insbesondere ab der zumeist in den „Einwanderungsländern“ geborenen sogenannten „zweiten Generation“, lehnt es ab, über ihren „Migrationshintergrund“ definiert zu werden. Pinar Atalay beispielsweise, seit Frühjahr 2014 Moderatorin der ARD-Tagesthemen, verwehrt sich gegen solche Zuschreibungen:

Eigentlich habe ich ja auch gar keine Migrationsgeschichte, ich stamme aus Lemgo [...]. Meine Eltern sind in den siebziger Jahren aus der Türkei nach Deutschland gekommen. Aber das Gewese zeigt mir doch, dass so eine Biografie anscheinend in den Köpfen der Leute noch keine Normalität ist. ${ }^{3}$

Geradezu skurril wird es, wenn sie - wie auch manche andere KollegInnen - quasi als Kompliment zu hören bekommt, dass sie doch sehr gut Deutsch spreche; und es dürften nicht nur Prominente wie Aylin Tezel oder Bülent Ceylan sein, die gar nicht ihre vermeintliche (türkische) Muttersprache verstehen, da schon immer Deutsch ihre Muttersprache war. ${ }^{4}$ Aller Unbill zum Trotz ist dennoch zu vermuten, dass ImmigrantInnen und ihren Nachfahren am besten dann gesellschaftlich ermöglicht wird, Schlüsselpositionen zu erlangen und damit Vorbilder, positive role models zu sein, wenn sie nicht über ihr ImmigrantInnen-Sein bzw. als ImmigrantInnen definiert werden. Dieses Ideal scheitert jedoch noch oft an den

3 Siehe Internetquellen: Karimian 2014.

4 Vgl. Internetquellen: Hukuki.net. 
tatsächlichen Verhältnissen, Wahrnehmungen und Zuschreibungen seitens Mitgliedern der Mehrheitsgesellschaft.

Aufschlussreich sind auch bevorzugte Eigenbezeichnungen mancher Immigrantengemeinschaften hinsichtlich ihres religiös konnotierten Selbstverständnisses. In europäische Länder eingewanderte afrikanische ChristInnen etwa möchten ihre Gemeinden nicht mehr als „Migrationskirchen“ rubriziert haben. Sie bevorzugen Bezeichnungen, die ihre internationalen und globalen Verflechtungen und damit bestenfalls einen transmigratorischen Aspekt in den Vordergrund stellen - so etwa die Christian Church Outreach Mission International mit Hauptsitz in Hamburg oder die Church of Pentecost International mit ihren National Headquarters ebenfalls in Hamburg. ${ }^{5}$ Ein mehrjähriges Forschungsprojekt unter afrikanischen „Migrations“-Kirchen kam jedenfalls zu dem eindeutigen Schluss, dass auf Begriffe wie „afrikanische Diaspora“, ,,afrikanische Migrationskirchen “ $o$. ä. künftig zu verzichten ist, da ihnen inzwischen nicht einmal mehr ein belastbarer heuristischer Wert zukommt. Dieser Befund erklärte sich nicht etwa aus einer unreflektierten Nostrifizierung der emischen Perspektive. Vielmehr ist neben Einwänden wie denen der Assoziation der Begrifflichkeit mit problematischen Zuschreibungen und impliziten Vorfestlegungen, perspektivischen Verzerrungen beim Blick auf das Forschungsfeld, Ausblendung des Selbstverständnisses und der Identitätspositionierungen der Befragten etc. insbesondere mit Blick auf VertreterInnen der jungen Generation und des weiteren Umfelds der untersuchten Kirchen nicht mehr zu rechtfertigen, mit diesen Begrifflichkeiten zu operieren. ${ }^{6}$

Dieser Punkt leitet zum zweiten anzusprechenden Thema über, dem der gesellschaftlichen und medialen Wahrnehmung von immigrierten, als fremd kategorisierten Religionen in westeuropäischen Gesellschaften: Politiker und Behördenvertreter reden zumeist über ImmigrantInnen und ihre Religionen und die Notwendigkeit der Anpassung. Wichtiger wäre jedoch, verstärkt mit religiösen Vertretern und Vertreterinnen zu sprechen. Ihre Perspektive zu Religionsausübung und benötigter Infrastruktur gilt es im direkten Austausch kennenzulernen. Insoweit sind die Initiativen zu einem offiziellen Dialog mit dem Islam, wie er 2006 erstmals vom früheren Innenminister Wolfgang Schäuble in Deutschland initiiert wurde und in die Gründung der „Deutschen Islamkonferenz" mündete, ${ }^{7}$ oder die österreichische Initiative des ehemaligen Staatssekretärs Sebastian Kurz zu einem „Dialogforum Islam“ im Jahr 2012 ausdrücklich zu begrüßen. ${ }^{8}$ Hier kann ein langfristig angelegter Dialog erfolgen, der es ermöglicht, mit den Betroffenen

5 Vgl. Internetquellen: Church of Pentecost International Germany.

6 Aus dem Anschlussbericht des von der Thyssen-Stiftung 2011-2014 geförderten Projekts (Klaus Hock, unveröffentlichter Abschlussbericht, 29.01.2016).

7 Vgl. Internetquellen: Deutsche Islam-Konferenz, Webseite; sowie Busch / Goltz 2011.

8 Vgl. Internetquellen: Bundesministerium für Inneres 2013. 
selbst bindende Absprachen über das Vorgehen bei der Integration zu treffen, drängende Probleme zu thematisieren und eine gemeinsame Evaluation der eingeleiteten Maßnahmen vorzunehmen. ${ }^{9}$

Zugleich bestehen jedoch auf Seiten vieler religiöser Immigrantengemeinschaften eklatante Defizite im Bereich der eigenen Interessenvertretung. Dies kann gleichermaßen für lokale religiöse Gemeinschaften wie für Dachverbände zutreffen. Gerade die oft fehlende Professionalisierung beim Agieren im öffentlichen Bereich, bedingt durch geringe Personal- und Wissensressourcen, führt hier zu mangelhafter Selbstvertretung gegenüber Behörden, Medien und Politik. Ansätze und Initiativen zur Qualifizierung von religiösen Leitern und Vorstandspersonal existieren zwar, können andererseits von diesen aufgrund der Überlastung durch Lohnarbeit und Leitungsfunktionen nur zum Teil wahrgenommen werden. Zudem sind solche Ansätze zur Qualifizierung nicht ganz unumstritten: Beispielsweise wird die gegenwärtige Einrichtung eines „Schweizer Zentrums für Islam und Gesellschaft“ an der Universität Fribourg von nationalkonservativer Seite vehement bekämpft und diffamiert. ${ }^{10}$ Die ,,theologischreligiöse Ausbildung“von Imamen sei nicht Aufgabe des Staates, sei zu teuer und letztlich, so die populistische Schweizerische Volkspartei, eine symbolische Anerkennung von radikalem Islam und islamischen Extremisten. ${ }^{11}$ Islam, muslimische Immigranten und Neubürger werden weiterhin von traditionalistischen, nationalkonservativen Kreisen als nicht zugehörig zur Schweiz deklariert und mit Stereotypen diffamiert. Auch in anderen Ländern finden sich analoge Äußerungen seitens populistischer rechtskonservativer Parteien wie der „Freiheitlichen Partei Österreichs“ (FPÖ) oder der „Alternative für Deutschland“ (AfD).

Im Vergleich mit der Schweiz stellt sich die Rechtslage für die muslimischen Gemeinschaften in Österreich insofern etwas besser dar, als eine offizielle Vertretung gegenüber dem Staat mit der Islamischen Glaubensgemeinschaft in Österreich (IGGiÖ) bereits seit 1912 gegeben ist ${ }^{12}$ und das neue Islamgesetz von 2015 die Position der Glaubensgemeinschaft gegenüber den Verbänden erheblich stärkt. ${ }^{13}$ Allerdings führen Spannungen zwischen den verschiedenen Dachver-

9 Allerdings sollte der Dialog über Integrationsmaßnahmen nicht nur auf muslimische ImmigrantInnen beschränkt werden. Mindestens ebenso wichtig ist der Dialog mit orthodoxen Einwanderern aus Südosteuropa, jüdischen MigrantInnen aus Russland oder katholischen MigrantInnen aus Lateinamerika, Afrika und Asien, die bisher in der Integrationsdebatte kaum berücksichtigt werden. Vgl. Polak / Reiss 2015.

10 Vgl. zum neuen Zentrum die Webseite und die Stellungnahme des Rektorats auf die Angriffe von Seiten der SVP (Internetquellen: Universität Freiburg).

11 Vgl. Internetquellen: Das Freiburger Bündnis.

12 Vgl. Heine / Lohlker / Potz 2012.

13 Eine der Grundideen des neuen Islamgesetzes von 2015 ist es, die Moscheegemeinden der verschiedenen Dachverbände in die Islamische Glaubensgemeinschaft in 
bänden und der IGGiÖ auch hier immer wieder zu unerquicklichen Konflikten, die manche Entwicklung behindern.

Als gesellschaftspolitische Entwicklung erwarten wir hier eine Weichenstellung, die auch für Forschungen zum Bereich „Religion und Migration“ wichtig ist: Entweder ermöglichen politische Entscheidungen eine verstärkte Öffnung zu und Anerkennung von ImmigrantInnen sowie ihren Religionen und ebnen damit den Weg zu gesellschaftlicher Teilhabe, Prestige und Wertschätzung. Möglicherweise wird dieser Weg gerade in Deutschland mit der Einrichtung der Zentren für Islamische Theologie an verschiedenen Universitäten bestritten, deren fortgesetzte Förderung auf weitere fünf Jahre durch das Bundesministerium für Bildung und Forschung vor einigen Monaten beschlossen wurde. ${ }^{14}$ Die andere Möglichkeit - die der Verweigerung und Ausgrenzung - droht in der Schweiz. Die Bekämpfung des neuen „Schweizer Zentrum für Islam und Gesellschaft“ durch die „Schweizerische Volkspartei“ (SVP) und der weitgehende Stillstand auf kantonalen Regierungsebenen, die rechtliche Anerkennung nicht-christlicher Religionen zu ermöglichen, sind hier sprechende Indizien.

Mit Blick auf die Frage der Wahrnehmung der religiösen Orientierung von ImmigrantInnen spielen die neuen sozialen Medien eine durchgängig ambivalente Rolle. Auf Seiten der Eingewanderten - seien sie nun MuslimInnen, ChristInnen oder Anhänger anderer Religionen - ermöglichen sie die Infragestellung traditioneller Autoritäten und bewirken damit Emanzipationsprozesse, die für die Interaktion mit der oftmals säkularisierten Residenzgesellschaft neue Verständnishorizonte eröffnet. Andererseits forcieren sie die Entstehung paralleler Kommunikationsräume, die Gefahr laufen können, sich diesen notwendigen Interaktionsprozessen zu entziehen. Auf Seiten der Residenzgesellschaft wiederum können die neuen Medien dazu beitragen, die bunte religiöse Vielfalt und Uneinheitlichkeit „fremder“ Religionen vor Augen zu führen und falsche Vorstellungen über „den Islam“ oder „die afrikanischen Christen“ korrigieren; andererseits dienen gerade auch sie als Katalysator beispielsweise islamfeindlicher Stereotypisierungen und Vorurteilspflege. Den destruktiven Momenten der neuen Medien ${ }^{15}$ wird auf Dauer nur mit dem langen Atem eines bewährten Qualitätsjournalismus und einer praxis- und öffentlichkeitsorientierten Forschung beizukommen sein. ${ }^{16}$

Österreich als Kultusgemeinden zu integrieren und ausländische Förderung zu unterbinden. 14 Vgl. Internetquellen: Bundesministerium für Bildung und Forschung.

15 Auch die in Deutschland aufgekommene „PEGIDA“-Bewegung (,Patriotische Europäer Gegen die Islamisierung Des Abendlandes") mit ihrer Hetze gegen die als „Lügenpresse“ oder „Systemmedien“ beschimpften Medien bezieht sich auf in den neuen Medien kursierende Informationen, die oftmals aus bewusst lancierten Falschmeldungen bestehen.

16 In den letzten Jahren hat es in dieser Hinsicht eine Reihe neuer Initiativen und Publikationen gegeben; vgl. etwa Klöcker / Tworuschka 2008, die von Tworuschka be- 


\section{Herausforderungen für Kirchen und Religionsgemeinschaften}

Herausforderungen für christliche Kirchen und die vielen alten und neuen weiteren Religionsgemeinschaften ergeben sich aufgrund äußerer, demnach gesellschaftlicher sowie innerer, also eigenverantworteter Entwicklungen und Konstellationen. Wir kommen hiermit auf die Metaphorik von „tragenden Netzen und schwankenden Böden“ im Titel dieses Beitrags zu sprechen. Auch hier sollen lediglich zwei Hauptpunkte mit jeweiligen Unteraspekten benannt werden.

Die einst mitgliederdominanten christlichen Volkskirchen sehen sich einem kontinuierlichen, teils dramatischen Mitgliederschwund ausgesetzt. Der Mitgliederschwund zwingt zur Zusammenlegung von Pfarreibezirken, zur Ausdehnung von Pastoralräumen und größerer Distanz zwischen Kirchenmitgliedern und Priestern oder PfarrerInnen. Schwindende Mitgliederzahlen führen zu weniger verfügbaren Ressourcen und zur Notwendigkeit der Reorganisation von Personal und Angeboten. In der Schweiz spricht man hier euphemistisch von „Optimierung“. Folgen hat dies im Sinne „schwankender Böden“ auf wenigstens zwei, sicherlich mehr Ebenen:

„Optimiert“ werden angesichts schwindender Ressourcen nicht zuletzt auch Leistungen im kirchlichen Sozialbereich wie Beratung, Hilfe und Unterstützung. Solche „tragenden Netze“ für Notleidende und ImmigrantInnen werden löchriger. Gesamtgesellschaftlich stellt sich damit verstärkt das Problem, welche Institutionen in der Zivilgesellschaft diesen notwendigen Sozialbereich übernehmen werden, wer Not- und Krisenfälle hilft abzufedern. Kommt es hier zu einer verstärkten kostenpflichtigen Privatisierung entsprechender Sozialdienste, zu einem stärkeren Engagement des Staats, oder schlicht zu einem Abbau solcher Sozialleistungen?

In Österreich ist insbesondere im Bereich der religiösen Betreuung in staatlichen Institutionen deutlich eine Veränderung dahingehend feststellbar, dass Bereiche, die bisher der Staat selbst als seine Aufgabe gesehen hatte, nunmehr verstärkt den Religionsgemeinschaften aufgebürdet werden. Orthodoxe, muslimische und jüdische Seelsorger sind in den neu abgeschlossenen Verträgen im Heer, im Gefängnis oder anderen Anstalten nicht mehr Angestellte des Staates. Vielmehr erhalten die Religionsgemeinschaften nur noch eine geringfügige pauschale Aufwandsentschädigung für ihre Tätigkeit. Dies hat zur Folge, dass die finanzielle Last von den Religionsgemeinschaften getragen werden muss, die Betreuer schlechter in die Institutionen eingebunden sind und eine De-Professionalisierung der Beratungstätigkeit stattfindet. Nicht zuletzt kommt es auch zur Konkurrenz mit und zur Benachteiligung gegenüber den traditionell etablierten christlichen Kirchen, die versuchen - bei aller Offenheit gegenüber den neuen

gründete Reihe „Studien und Dokumentationen zur Praktischen Religionswissenschaft“ (LIT) sowie die von Wolfram Reiss und Ulrike Bechmann herausgegebene Reihe „Anwendungsorientierte Religionswissenschaft“ (Tectum). 
Religionsgemeinschaften - ihre Privilegien (staatliche Subventionierung von Stellen, Räumen, Büros, Kapellen, Sachmitteln) so lange wie möglich zu erhalten. ${ }^{17}$

Angesichts der Tatsache, dass der bislang Sicherheit verbürgende, fürsorgende Sozialstaat seine Leistungen in den letzten Jahren sukzessive zurückgeschraubt hat, werden neue, von der Zivilgesellschaft getragene Initiativen, Aktions- und Organisationsformen umso dringlicher. Für die Kirchen ergibt sich zugleich die Herausforderung, dass ihr gesamtgesellschaftlich positives Image, das auf ihrem sozialen Engagement für Benachteiligte beruht, schwindet. ${ }^{18}$ Damit schwinden jedoch in Augen vieler auch die Bedeutung und die Legitimität der Kirchen für die Gesellschaft. $^{19}$

Hat in diesem Aspekt der Boden schon zu schwanken begonnen, so ebenso hinsichtlich der Frage, welcher gesellschaftliche Einfluss den Volks- oder Landeskirchen zustehe. Lange Zeit als moralische Autorität und ethische Instanz in staatlichen Kommissionen und öffentlich-rechtlichen Medienanstalten vertreten, kritisieren Humanistenverbände und andere diese weithin als Selbstverständlichkeit betrachtete Besetzung einflussreicher Positionen durch die Kirchen. Kirchliche Vertreter werden - gerade nach diversen Skandalen - immer weniger als ,gutes Gewissen der Gesellschaft“ wahrgenommen, sondern stärker als parteiische Interessensvertreter mit religiösen Partikularansichten. Was legitimiert dann jedoch die Besetzung einflussreicher Positionen, zumal die Möglichkeit staatsrechtlicher Anerkennung zunehmend nicht mehr nur auf christliche Konfessionen beschränkt bleiben wird? Dies stellt eine kritische Herausforderung für viele christliche Kirchen im politischen Feld dar. ${ }^{20}$

Die christlichen Kirchen werden langfristig nicht umhinkommen, sich auch im diakonischen Bereich grundlegend zu restrukturieren sowie im Zuge dieser Transformationsprozesse als Akteure neben anderen, z. B. im Entstehen begriffenen islamischen Wohlfahrtsverbänden zu verstehen und gemeinsam mit diesen zu agieren. Neben der notwendigen Reorganisation mit Blick auf Personal und Dienstleistungen könnten aus der Neuausrichtung parochialer Ressourcen Impulse für das Entstehen neuer, durchaus recht tragfähiger Gestalten zivilgesellschaftlichen Engagements ausgehen. Die Tatsache des zunehmenden Verfalls kirchlicher Hegemonialität wäre so durchaus positiv zu wenden: Mit der Anerkennung religiöser Polyphonie und „konzertierten Aktionen“ gemeinsam mit religiösen oder säkularen nichtchristlichen Akteuren könnte die moralische Autorität der Kirchen neue Anerkennung finden. Auf diese Weise wäre es zudem möglich, die Integrationspotenziale von Religionsgemeinschaften generell zu

17 Vgl. Internetquellen: Reiss 2016.

18 Für die Schweiz vgl. Internetquellen: Stolz / Könemann et al. 2011, S. 27 f.

19 Vgl. Internetquellen: Stolz / Chaves et al., S. 37.

20 Zur Debatte um die staatliche Anerkennung von Religionsgemeinschaften siehe jüngst Pahud de Mortanges 2015. 
stärken, indem etwa nach gemeinsamen, Religionen übergreifenden Strategien der Inklusion von ImmigrantInnnen gesucht wird - insbesondere im sozialen und politischen Bereich, aber durchaus auch darüber hinausgehend auf kulturellem Gebiet, dem als gemeinsam zu gestaltendes Feld nicht zu unterschätzende Bedeutung zukommt.

Welche Herausforderungen zeigen sich für Religionsgemeinschaften von ImmigrantInnen? Kulturelle, religiöse und politische Institutionen und Gemeinschaften sind in den westeuropäischen Ländern mittlerweile fest etabliert. Im Bereich der Religionen wird dies nicht zuletzt sichtbar an mittlerweile zahlreichen repräsentativen Sakralbauten, die neben Kirchtürmen und Kirchenbauten den öffentlichen Raum einer Stadt oder Landgemeinde mitbestimmen. ${ }^{21}$ Konflikte und Kontroversen um diese Neubauten, gerade im Fall von Moscheen und Minaretten, dürften bei mangelnder Kommunikation des Vorhabens seitens der Bauherren gegenüber den Anwohnern und angesichts des Erstarkens rechtsradikaler Parteien auch weiterhin immer wieder aufflammen. Doch mit der heranwachsenden zweiten Generation ist auch ein besseres Verständnis vorhanden, wie Prozesse vorausschauender Information, Gewinnung lokaler Partner und Unterstützer sowie Themen der Architektursprache zu handhaben sind. ${ }^{22}$

Zuerst einmal sind religiöse Andachtsstätten Orte für Gebet, religiöse Versammlung, Ritual und das Feiern religiöser Feste. Religiöse Versammlungsstätten fungieren für ImmigrantInnen aber oft auch als Orte der Zuflucht und „,kleine Heimat in der Fremde“. Sie schaffen Vertrauen und Beziehungen zwischen den jeweiligen Gläubigen, bieten moralisch-ethische Orientierung und religiös-kulturelle Selbstvergewisserung. So berichtet beispielsweise ein Flüchtling aus dem Kongo, dass er in einer afrikanischen Kirche neue Freunde, Halt und Kraft gefunden habe. Er sagt: „Die Kirche ist für mich auch heute noch wie eine Tankstelle, [...] ich gehe hin und tanke auf “23. Das Beispiel zeigt, dass Kirchen, Moscheen und Tempel über ihre religiösen Kernaufgaben hinaus auch soziale Treffpunkte sind. Hier werden Informationen zu sozialen, kulturellen, beruflichen und informellen Belangen ausgetauscht. Die bei vielen ImmigrantInnengruppen zumeist sprachlich-kulturell organisierten religiösen Stätten bilden ein

21 Vgl. die Website Moscheesuche (Internetquellen: Moscheesuche). In Österreich gibt es nur 4 äußerlich erkennbare Moscheen. Vgl. Schuller 2013. Für die Schweiz siehe die Dokumentation „Kuppel - Tempel - Minarett“ (vgl. Internetquellen: Universität Luzern), die alle seit 1945 neu erbauten Sakralbauten von ImmigrantInnen erfasst hat; siehe außerdem Internetquellen: Baumann / Tunger-Zanetti 2011.

22 Zu Konflikten um Moschee- und Minarettbauten existieren zahlreiche Studien für Deutschland, Österreich, die Schweiz und andere europäische Staaten. Exemplarisch seien genannt Hüttermann 2006; vgl. Internetquellen: Allievi 2009; Beinhauer-Köhler / Leggewie 2009; Bernhardt / Fürlinger 2015; Fürlinger 2015 sowie Baumann / TungerZanetti 2016.

23 Siehe Internetquellen: Jäggi / Schär 2009, S. 10. 
tragendes Netz, das ImmigrantInnen Rückhalt und Selbstvertrauen gibt, Alltagswissen vermittelt und bei Bedarf Beratung und Hilfe zur Verfügung stellt. Der Soziologe Georg Elwert sprach von einer „Binnenintegration“, einer Einbindung vorerst in die eigene religiös-kulturelle Gemeinschaft. ${ }^{24}$

Wie neuere Forschungen zeigen, gehen Angebote vieler religiöser Gemeinschaften von ImmigrantInnen jedoch weit über die Einbindung in die eigene Gruppe hinaus. Moscheen, Kirchen und Pagoden haben sich anders als im Herkunftsland oftmals zu „multifunktionalen Dienstleistungszentren“ entwickelt. Beispielsweise bieten viele Moscheevereine über das gemeinsame Gebet und die Predigt am Freitag hinaus soziale Angebote wie Jugendarbeit, Beratungen für Kindererziehung, Nachhilfe, Weiterbildungs- und Freizeitangebote sowie die Organisation von Sport und Ausflügen an. ${ }^{25}$ Analoges gilt für Kirchen und Gemeinden, die aus der Initiative christlicher Einwanderer entstanden sind. Hatte sich ihr soziales Engagement ursprünglich auf Nothilfe bei aufenthaltsrechtlichen oder finanziellen Problemen konzentriert, gehen die inzwischen angebotenen Dienstleistungen weit über solche karitative Sofortmaßnahmen hinaus und umfassen ein äußerst breites Portfolio von Unterstützungs-, Fortbildungs- und Vernetzungsmaßnahmen. Nachhilfeunterricht und Weiterbildung beispielsweise sind in diesem Zusammenhang besonders wichtig, um bessere Möglichkeiten zur gesellschaftlichen Teilhabe im beruflichen und sozialen Bereich zu eröffnen. Solche Angebote werden mitunter gerade in der Moschee oder im vernetzten Rahmen wahrgenommen, da dies ein geschützter und vertrauter Raum ist, der auch Frauen eine Teilnahme ermöglicht. Voraussetzung für Frauen, die Angebote wahrzunehmen, sind jedoch eigene Räumlichkeiten. Interessanterweise ermöglichen gerade Moschee-Neubauten, die mehr Platz bieten als Hinterhofmoscheen, solche eigenen Räume und tragen damit zum Mitwirken von Frauen bei. ${ }^{26}$

Die Leistungen von Religionsgemeinschaften sollen jedoch nicht einseitig nur positiv benannt sein. Es zeigen sich auch Grenzen. So können etwa bei religiösen ImmigrantInnen frühere Machtstrukturen, Altershierarchien und gegebenenfalls Kastengrenzen und tribale Ehrvorstellungen durch traditionell tonangebende Personen fortgeschrieben werden. Beispielsweise werden Frauen und Jugendlichen in den religiösen Institutionen häufig lediglich begrenzte Mitsprache, Räume und Einflussmöglichkeiten zugestanden. Oft besteht ein hoher Konformitätsdruck bei Geschlechterrollen und Entfaltungsräume können insbesondere für Frauen in Bezug auf Kleidung, Freizeitverhalten und Wahl des Ehepartners eng sein. Religionsgemeinschaften sind hier gefordert, eine stärkere Mitgestaltung durch viele zu eröffnen und gerade Frauen und Heranwachsende einzubeziehen.

24 Vgl. Elwert 1982, S. 717-723. Zu neueren Forschungen siehe Weiss / Thränhardt 2005; Nagel 2013 sowie Baumann 2015.

25 Vgl. Behloul / Lathion 2007, S. 201.

26 Vgl. Stöckli 2014. Zu den zivilgesellschaftlichen Potenzialen siehe Nagel 2015; Sachverständigenrat 2016, S. 50-61, sowie Arens / Baumann / Liedhegener 2016. 
Die Herausforderung für die Religionsforschung wiederum besteht darin, diese Formen negativen Sozialkapitals nicht auszublenden, sondern mit kritischem Blick ebenso wie die von den betreffenden Religionsgemeinschaften ausgehenden positiven Impulse wahrzunehmen und zu erforschen. ${ }^{27}$

Dabei ist äußerste Sorgfalt anzumahnen und vorschnelle Schlüsse sind $\mathrm{zu}$ vermeiden. So wurde etwa vor einigen Jahren in einer Studie ein Zusammenhang zwischen erhöhter islamischer Religiosität und der Gewaltbereitschaft muslimischer Jugendlicher konstatiert. Konkret hieß es, „dass eine hohe Religiosität die Integration von jugendlichen Migranten nicht behindert; dies gilt allerdings erneut nicht für muslimische Migranten “28. Bei genauerer Betrachtung zeigt sich allerdings, dass nicht der Islam die Ursache für die Gewaltbereitschaft junger Muslime ist. Einen gewissen Anteil mögen vorübergehend aus dem Ausland eingereiste Imame haben, ${ }^{29}$ die ein konservatives patriarchalisches Weltbild vermitteln, wie die Autoren der Studie vermitteln. Die Wirklichkeit dürfte jedoch noch komplexer sein, wenn mit Blick auf die Religiosität junger Muslime zugleich festzustellen ist, dass die meisten von ihnen kaum nennenswertes Grundwissen über den Islam haben und Religion lediglich einen rein äußerlichen Bezugspunkt darstellt. ${ }^{30}$

In der Frage der Herausforderungen für Religionsgemeinschaften durch Migration sind aber nicht nur die „Migrationsgemeinden“ gefordert, sondern auch die etablierten Religionsgemeinschaften. Begegnungen mit nahe gelegenen Moscheegemeinden, mit afrikanischen Kirchen oder auch mit orthodoxen Gemeinden kann es katholischen und evangelischen Gemeinden ermöglichen, neue Impulse in der eigenen Gemeinde zu setzen und zugleich Kontakte mit neuen Partnern auf Gemeindeebene aufzunehmen, in denen sich die gesellschaftlichen Veränderungen ausschnitthaft, aber exemplarisch spiegeln. „Ökumene“ würde bedeuten, dass alle religiösen Vertreter in einer bestimmten Region einander begegnen, sich austauschen und lernen, gegebenenfalls auch gemeinsame Interessen dem Staat gegenüber zu formulieren. In Wien finden solche vom Magistrat geförderten interreligiösen Begegnungen bereits seit einigen Jahren statt. ${ }^{31}$

27 Auf die Notwendigkeit verstärkter empirischer Forschungen verwies schon früher Traunmüller 2008, S. 3, und ders. 2013.

28 Siehe Internetquellen: Baier et al. 2010, S. 9.

29 Vgl. Ceylan 2010.

30 Auf diesen Zusammenhang hat Rauf Ceylan u. a. bereits in seiner vor zehn Jahren erschienenen Dissertation aufmerksam gemacht (Ceylan 2006). Offensichtlich herrscht eine Diskrepanz zwischen Jugendlichen, die sich als hochreligiös betrachten, aber einen faktisch religionsfernen Lebensstil pflegen und über ihre Religion nichts wissen und Jugendlichen, die in muslimischen Gemeinden engagiert sind und aufgrund der dort herrschenden sozialen Kontrolle weniger kriminell und gewalttätig werden.

Vgl. Internetquellen: Magistrat der Stadt Wien. 
Mit Blick auf das Heranwachsen der zweiten, teils dritten Generation stellt sich die Frage, wie sie die religiöse Tradition ihrer Eltern interpretieren werden. Angesichts starker Prozesse von Individualisierung, Privatisierung und dem Hinterfragen religiöser Ideen und Rituale zeichnet sich bei nicht wenigen eine Distanz zu überkommener Religion ab. ${ }^{32}$ Die Abkehr von den Institutionen der ersten Generation führt teilweise zur Gründung eigenverantworteter ,tragender Netze" durch die jungen Erwachsenen, seien dies neue Sozialformen wie moschee-unabhängige muslimische Jugendgruppen, Chat-Foren im Internet oder religions- und herkunftsübergreifende Treffen wie die Wake-Up-Gruppen des vietnamesischen Mönchs Thich Nhat Hanh. ${ }^{33}$ Für die etablierten religiösen Immigrantengemeinschaften sowie ihre Imame und Priester stellt sich die Herausforderung, belastbare Antworten auf die Frage zu finden, welche Formen und Änderungen notwendig sind, um die Jugend an Moscheen, Tempel und Kirchen zu binden. Andererseits sind dieser Tendenz zum Auszug der zweiten bzw. dritten Generation aus hergebrachten Institutionen aufgrund von Individualisierung, Privatisierung und Konsumprimat durchaus auch positive Aspekte abzugewinnen. Diese wären etwa darin zu sehen, dass sich zwischen den neuen, hinzugekommenen und den alten, in der Residenzgesellschaft verankerten Religionsgemeinschaften neue Gesprächsräume eröffnen könnten - denn beide haben mit Blick auf den Trend hin zu einem „Auszug“ der Jugendlichen aus ihrer religiösen Heimat mit ähnlichen Problemen zu tun.

Eine zentrale Herausforderung für ImmigrantInnen wird es wohl in der $\mathrm{Zu}$ kunft sein, Möglichkeiten zur Wahrung von Sprache und kultureller Identität zu schaffen und darin auch von staatlicher bzw. kommunaler Seite unterstützt zu werden. Die zweite und dritte Generation sollte ermutigt werden, nicht nur Deutsch zu lernen, sondern mindestens zwei Sprachen zu beherrschen - nämlich die ihrer eingewanderten Eltern und Großeltern, und die der Residenzgesellschaft, also beispielsweise Arabisch und Deutsch, Türkisch und Deutsch, Urdu und Deutsch usw. Religiöse Orte sollten sich zunehmend zu Orten verwandeln, in denen nicht nur das Bedürfnis nach religiöser Heimat gestillt wird, sondern wo auch Begegnungen mit der Mehrheitskultur stattfinden. Dies ist aber nicht nur eine Aufgabe für ImmigrantInnengemeinden, sondern für diese und gemeinsam mit Kommunen sowie etablierten Religionsgemeinschaften.

Von zentraler Bedeutung ist, dass künftig Leiter von Immigrationsgemeinden vornehmlich in Europa ihre Ausbildung erfahren. Insoweit sind die derzeitigen Bemühungen um eine Begründung von theologischen Ausbildungsstellen für Imame, SeelsorgerInnen und ReligionslehrerInnen oder auch um orthodoxe theologische Lehrstühle für Priester und ReligionspädagogInnen an europäischen

32 Vgl. exemplarisch Müller 2013 und Schlieter / Kind / Lauer 2014.

33 Zu moschee-unabhängigen muslimischen Jugendgruppen siehe Endres et al. 2013. Zu Chat-Foren siehe Eickelman / Anderson 2003; Engelmann 2010. Zu den Wake-UpGruppen vgl. Internetquellen: Wake Up International. 
Hochschulen von zentraler Bedeutung. Allerdings wird es auch eine Herausforderung darstellen, zugleich an die traditionellen Bildungseinrichtungen z. B. in der Türkei, in Ägypten oder in Persien bzw. in Griechenland, Serbien, Rumänien und Bulgarien anzuknüpfen, um die Anschlussfähigkeit der islamischen bzw. orthodoxen Ausbildung zu gewährleisten.

\section{Neue Forschungsfragen im Kontext gesellschaftspolitischer und religionsgemeinschaftlicher Entwicklungen}

Wie kann in der medialen Öffentlichkeit eine sachlichere und weniger vorurteilsbelastete Darstellung insbesondere des Islam und der MuslimInnen, aber auch beispielsweise von AfrikanerInnen und ihrem oftmals charismatisch geprägtem Christentum sowie aller anderen Religionsformen von ImmigrantInnen möglich werden? Diese Frage ist zwar vornehmlich eine Herausforderung für die Politik und die Medien, aber Forschung kann Medien analysieren und feststellen, inwieweit Vorurteile und verzerrte Darstellungen über ImmigrantInnen vermittelt werden. Möglicherweise sollte auch stärker in Richtung auf eine pro-aktive Haltung und nicht nur reaktive Haltung der Religionswissenschaft hingearbeitet werden. Fakt ist jedenfalls, dass unter den selbsternannten oder von Medien dazu gekürten „Religionsexperten“ sich bisher nur in relativ bescheidenem Maße Religionswissenschaftler finden bzw. Gehör finden. Hier wäre ein stärkerer gesellschaftlicher Einfluss der Religionswissenschaft wünschenswert, allerdings muss dies einhergehen mit einem gründlichen Überdenken der wissenschaftstheoretischen Grundlagen für solch ein Engagement. ${ }^{34}$

Darauf baut die Frage auf, wo sich Ansätze zur Entdramatisierung des pejorativen Islam-Diskurses zeigen. Ansätze zeigen sich etwa bei nicht-konfliktiven Moscheebauprojekten, wie sie in Penzberg in Oberbayern und in Duisburg im Ruhrgebiet glückten. ${ }^{35}$ Eine frühe Kommunikation, die starke lokale Einbindung, die Beteiligung von vielen Gemeindemitgliedern und ein offener, gelehrter und kultursensibler Imam sind wichtige Voraussetzungen, um Vorbehalte in der lokalen Bevölkerung und Politik abzubauen. ${ }^{36}$

Weiterhin stellt sich uns die Frage, wie die Integrationspotenziale von Religionsgemeinschaften, insbesondere von ImmigrantInnengemeinschaften, gesellschaftspolitisch stärker zur Geltung gebracht werden können. Im politischen Diskurs, gerade in konservativen Parteien, herrscht weiterhin ein Bild von ImmigrantInnengemeinschaften vor, das diese als sich abschottende Parallelgesellschaften zeichnet. In der Realität, und wie empirische Forschungen zeigen, finden wir jedoch vielfach integrative Angebote und unterstützende Netze, die

\footnotetext{
34 Vgl. Reiss 2012.

35 Vgl. Jasarevic 2009 und Internetquellen: Jenkner 2008.

36 Vgl. dazu u. a. Idriz 2010.
} 
die Gläubigen tragen, einbinden und ermutigen. Können diese Dienstleistungen von ImmigrantInnengemeinschaften womöglich teilweise die absehbar verringerten Unterstützungsleistungen christlicher Kirchen im Sozialbereich kompensieren, oder kann eine koordinierte Betreuung aufgebaut werden, die nicht nur durch katholische und evangelische Kirchen getragen wird, sondern durch ganz verschiedene christliche und nicht-christliche Religionsgemeinschaften? Eine gesellschaftliche Wahrnehmung und Anerkennung der zumeist ehrenamtlich geleisteten Dienste in den Gemeinschaften wäre hierfür eine wichtige Voraussetzung. ${ }^{37}$ Religions- und sozialwissenschaftliche Forschungen können hier einen großen Beitrag leisten, indem sie durch empirische Studien darauf hinweisen, wo Integrationspotentiale vorhanden sind, wo Integration gelingt oder misslingt und welche Faktoren dabei eine Rolle spielen. Dies kann indirekt eine wichtige Hilfestellung für Politik und Religionsgemeinschaften darstellen, da damit Defizite aufzudecken oder umgekehrt Beispiele für best practices zu eruieren wären, die dann von den Verantwortlichen in der Politik und innerhalb der Religionsgemeinschaften gegebenenfalls aufgegriffen werden können.

Damit zusammen hängt die Frage, welche tradierten Vorstellungen und etablierten Machtstrukturen dafür mitverantwortlich sind, dass die vor 30 oder 50 Jahren von ImmigrantInnen mitgebrachten Religionen in der Öffentlichkeit mehrheitlich nicht als legitimer Teil der existierenden Religionsvielfalt eines Landes angesehen werden. Buddhisten, Hindus, Sikhs, orthodoxe oder afrikanische Christen und Muslime werden zumeist entweder als verklärt-exotisches oder aber als fremd-gefährliches Phänomen angesehen. Als der seinerzeitige deutsche Bundespräsident Christian Wulff im Jahr 2010 aussprach, was nicht mehr als die Feststellung einer gesellschaftlichen Realität war - „der Islam gehört inzwischen auch zu Deutschland“38 - gab es einen Aufschrei im konservativen Lager. Fünf Jahre danach wiederholte die deutsche Bundeskanzlerin Angela Merkel den Satz, ein Aufschrei blieb diesmal jedoch aus. Immerhin ein gutes Zeichen. Kurze Zeit später jedoch wird von rechtskonservativen und islamfeindlichen Kräften unter expliziter Bezugnahme auf diesen Satz in bewusster Provokation postuliert: „Der Islam gehört nicht zu Deutschland““39. Mehr denn je stellt sich vor dem Hintergrund dieser Entwicklungen auch und gerade für ReligionswissenschaftlerInnen die Aufgabe, darauf zu drängen, dass die gesamte religiöse und kulturelle Vielfalt,

37 Bei einem Seminar in Wien, bei dem über das Thema „Alter“ in einem sehr durchmischten Stadtteil Wiens geforscht wurde, kam heraus, dass z.B. muslimische Gemeindevorstände immens viele regelmäßige Besuche von älteren Gemeindemitgliedern durchführen. Allerdings wurde dies im Unterschied zu den etablierten christlichen Gemeinden bisher nie unter dem Aspekt einer aktiven „Altenarbeit“ betrachtet.

38 Siehe Internetquellen: Wulff 2010. Die Urheberschaft der kontrovers diskutierten Aussage wird jedoch dem damaligen Bundesinnenminister Wolfgang Schäuble beim ersten Treffen der Islamkonferenz 2006 zugeschrieben, vgl. Internetquellen: Detjen.

39 Siehe Internetquellen: Alternative für Deutschland, S. 34. 
die sehr stark insbesondere unsere Städte prägt, als ein selbstverständlicher Bestandteil unserer Kultur ins Bewusstsein gerückt werden sollte. ${ }^{40}$

Vermutlich verlieren Fragen von Zugehörigkeit, Fremdwahrnehmung und Anerkennung von immigrierten Religionen im Lauf der Zeit an Bedeutung. Wurden in den 1960ern und 1970ern noch sehr stark Italiener, Griechen und Spanier als Fremde wahrgenommen, so ist dies heute kaum noch der Fall. Nicht unwahrscheinlich ist auch, dass die gesellschaftlichen Entwicklungen von Individualisierung und Konsumorientierung, gerade auch bei der individuellen Nachfrage auf dem Religionsmarkt, ${ }^{41}$ sich ähnlich wie in den vergangenen fünf Jahrzehnten fortsetzen werden. Dies bedeutet ein anhaltendes Schrumpfen der Kirchen, eine weitergehende Zunahme des Anteils Religions-Ungebundener an der Gesamtbevölkerung und eine verstärkte Konsolidierung von ImmigrantInnenreligionen. Womöglich entsteht in verschiedenen europäischen Ländern ein Wettbewerbsmarkt von kleineren und größeren Denominationen und der Modus staatsrechtlicher Anerkennung wird ausgeweitet oder fällt gänzlich fort. ${ }^{42}$ Forschungsfragen können sich dann dem Freiwilligkeitscharakter der Denomination, dessen bereitgestellten „Heilsgütern“ und Dienstleistungen, möglichen Milieugründungen sowie den Werbe- und Aufmerksamkeitsstrategien von Denominationen widmen. ${ }^{43}$

Auf (religions)theoretischer und konzeptioneller Ebene bleibt die Frage, wie dichotomisierende, essentialisierende und zugleich auf undurchschaubare Weise hermetisch verschränkte Diskurse aufgebrochen werden können. Diese Diskurse reden immer noch einem Gegenüber von christlich geprägter moderner, säkularisierter Kultur und nichtchristlich geprägten rückständigen, mit Europa inkompatiblen religiösen Traditionen das Wort. Die besondere Aufgabe für die Forschung wird in diesem Zusammenhang auch darin bestehen, zur Klärung des Widerspruchs beizutragen, dass in wissenschaftlichen Diskursen über „Kulturbegegnung“ die Verwendung essentialisierender Kategorien und die Annahme kultureller Dichotomien weitgehend überwundenen ist, ${ }^{44}$ in öffentlichen Diskursen jedoch geradezu reüssiert.

40 Vgl. Baumann 2004; Reiss 2015, S. 145-149. Zudem gibt es mittlerweile zahlreiche Darstellungen zur religiösen Vielfalt im urbanen Raum, so etwa zu Basel, Berlin, Frankfurt/Main, Graz, Wien, Zürich und vielen anderen Städten; siehe die Zusammenstellung „Religionen vor Ort - Darstellungen und Untersuchungen“ bei REMID (vgl. Internetquellen: Religionswissenschaftlicher Medien- und Informationsdienst).

41 Vgl. Stolz 2014.

42 Zur Anerkennungsfrage im deutschsprachigen Bereich siehe Pahud de Mortanges 2015.

43 Zur religiösen Organisationsform der Denomination siehe klassisch Niebuhr 1971 sowie u. a. Scherer 1980; Liebman et al. 1988; Demerath III et al. 1998.

44 Vgl. etwa die Forschungen im Kontext des Graduiertenkollegs „Kulturkontakt und Wissenschaftsdiskurs“, die von der Einsicht ihren Ausgang nehmen: „Während ein 
Vor dem Hintergrund der hier aufgelisteten und sicherlich bei weitem nicht umfassenden Herausforderungen für die Religionsforschung stellt sich insbesondere mit Blick auf die Rolle der Religionsgemeinschaften in der Migrationsgesellschaft für alle religionsbezogenen Wissenschaften und darüber hinaus die fundamentale Frage: Welche säkulare Gesellschaft, welche Säkularität brauchen wir, damit über den Transmissionsriemen der tatsächlichen Anerkennung positiver Religionsfreiheit wirkliche Religionspluralität und damit auch die „neuen“, „dazugekommenen“ Religionen in unserer Gesellschaft Akzeptanz finden können?

\section{Schluss}

Die Religionsforschung hat in den vergangenen Jahren eine Reihe wichtiger Studien und Analysen zum Themenbereich Religion und Migration vorgelegt. Ein gesellschaftlicher „Impact“ der Forschungen kann jedoch nur im Zusammenspiel mit politischen, zivilgesellschaftlichen und umsetzungsorientierten Initiativen erfolgen. Erfreulicherweise sind einige der hier angesprochenen Herausforderungen auf konkreten Praxis- und Politikfeldern in Politik und Zivilgesellschaft aufgegriffen - oftmals gegen den Trend im öffentlichen Diskurs. So hat in Deutschland die Antidiskriminierungsstelle des Bundes 2016 zum ,,Themenjahr gegen Diskriminierung wegen der Religion oder Weltanschauung“ erklärt und unter dem Motto „Freier Glaube. Freies Denken. Gleiches Recht.“ eine Reihe von Publikationen herausgegeben, unter denen sich Forschungsergebnisse zur Frage der Akzeptanz religiöser und weltanschaulicher Vielfalt in Deutschland auf der Grundlage einer repräsentativen Studie ebenso finden wie rechtswissenschaftliche und sozialwissenschaftliche ${ }^{45}$ Expertisen zum Problem der „Diskriminierung aufgrund der islamischen Religionszugehörigkeit im Kontext Arbeitsleben“. Zusätzlich hat im Frühling 2016 der „Sachverständigenrat deutscher

z. T. vom Strukturalismus geprägtes, jedoch in Wirklichkeit auf archaische Vorstellungen von kultureller Differenz zurückgehendes dualistisches Wissenschaftsparadigma, das von Kulturen im Sinne von binären Oppositionen und unvereinbaren fundamentalen Gegensätzen spricht, in der populärwissenschaftlichen Forschung und im öffentlichen Diskurs weiterhin virulent ist, hat der internationale akademische Diskurs der letzten zwei bis drei Jahrzehnte eine deutliche Hinwendung zu komplexeren und dialogischeren Erklärungsmustern für das Phänomen ,Kulturkontakt" vollzogen.“ (Vgl. Internetquellen: Graduiertenkolleg „Kulturkontakt und Wissenschaftsdiskurs“ der Universität Rostock.) 45 Vgl. Internetquellen: Antidiskriminierungsstelle des Bundes 2016; Internetquellen: Frings 2010, sowie Internetquellen: Peuker. Auch in der Schweiz finden sich mit der Eidgenössischen Migrationskommission (vgl. Internetquellen: Eidgenössische Migrationskommission) und deren Publikationen sowie in Österreich mit der Gleichbehandlungsanwaltschaft (vgl. Internetquellen: Gleichbehandlungsanwaltschaft) entsprechende Fachstellen. 
Stiftungen für Integration und Migration“ (SVR) in seinem Jahresgutachten 2016 die religiöse Vielfalt im Einwanderungsland Deutschland untersucht. ${ }^{46}$ Ergebnis der differenzierten Studie ist unter anderen, „dass religiöse Gemeinschaften wichtig sind für aktives zivilgesellschaftliches Engagement und die damit verbundene Einbindung in soziale Netzwerke“47. Zugleich bestehe jedoch „hinsichtlich des Zusammenhangs von Religion und Integration eine deutliche Diskrepanz zwischen der gesellschaftlichen Diskussion über die Bedeutung von Religion und empirisch fundierten Belegen dazu“ sowie dass „die Bedeutung von Religion für gesellschaftliche Teilhabe allgemein überschätzt wird“48. Der Befund mahnt damit zu einer Entdramatisierung des Themas Religion und Migration, fordert jedoch zugleich weitere Studien, um die vielfältigen Sachverhalte und Zusammenhänge auf empirischer Grundlage differenziert zu erforschen.

\section{Literaturverzeichnis}

Arens, Edmund / Baumann, Martin / Liedhegener, Antonius: Integrationspotenziale von Religion. Baden-Baden 2016 (im Druck).

Baumann, Martin: „Kirchen, Moscheen, Synagogen - eine Stadt verträgt viele Religionen“, in: Jansen, Mechthild M. / Keval, Susanne (Hg.): Die multireligiöse Stadt. Religion, Migration und urbane Identität (= POLIS 40, Reihe der Hessischen Landeszentrale für politische Bildung). Frankfurt am Main 2004, S. 16-30; Wiederabdruck in: Amt für multikulturelle Angelegenheiten der Stadt Frankfurt am Main et al. (Hg.): Religion und Migration. Frankfurt am Main 2007, S. 69-83.

Baumann, Martin: „Von Gegenorten zu neuen Brücken- und Heimatorten: Moscheen, Tempel und Pagoden von Immigranten in der Schweiz“, in: Mohn, Jürgen / Hermann, Adrian (Hg.): Orte der Europäischen Religionsgeschichte. Würzburg 2015, S. 503-523.

Baumann, Martin / Tunger-Zanetti, Andreas: „Neue Sakralbauten in der Politik des Raums. Eine dimensionen-basierte Analyse der Errichtung religiöser Gebäude in der Schweiz“, in: Beinhauer-Köhler, Bärbel / Frank, Edith (Hg.): Religion, Raum, Natur: Religionswissenschaftliche Erkundungen. Münster 2016.

Behloul, Samuel-Martin / Lathion, Stéphane: „Muslime und Islam in der Schweiz: viele Gesichter einer Weltreligion“, in: Baumann, Martin / Stolz, Jörg (Hg.): Eine Schweiz viele Religionen. Risiken und Chancen des Zusammenlebens. Bielefeld 2007, S. 193207.

Beinhauer-Köhler, Bärbel / Leggewie, Claus: Moscheen in Deutschland. Religiöse Beheimatung und gesellschaftliche Herausforderung. München 2009.

Bernhardt, Reinhold / Fürlinger, Ernst (Hg.): Öffentliches Ärgernis? Moscheebaukonflikte in Deutschland, Österreich und der Schweiz. Zürich 2015.

46 Vgl. Internetquellen: Sachverständigenrat deutscher Stiftungen für Integration und Migration.

47 Ebd., S. 58.

48 Ebd., S. 61. 
Busch, Reinhard / Goltz, Gabriel: „Die Deutsche Islam Konferenz - Ein Übergangsformat für die Kommunikation zwischen Staat und Muslimen in Deutschland", in: Meyer, Hendrik / Schubert, Klaus (Hg.): Politik und Islam. Wiesbaden 2011, S. 29-46.

Ceylan, Rauf: Die Prediger des Islam. Imame - wer sie sind und was sie wirklich wollen. Freiburg im Breisgau 2010.

Ceylan, Rauf: Ethnische Kolonien. Entstehung, Funktion und Wandel am Beispiel türkischer Moscheen und Cafés. Wiesbaden 2006.

Demerath III, Nicholas J. et al. (eds.): Sacred Companies: Organizational Aspects of Religion and Religious Aspects of Organizations. Oxford 1998.

Eickelman, Dale F. / Anderson, Jon W.: New Media in the Muslim World. The emerging Public Sphere. Bloomington ${ }^{2} 2003$.

Elsas, Christoph: Religionsgeschichte Europas. Religiöses Leben von der Vorgeschichte bis zur Gegenwart. Darmstadt 2002.

Elwert, Georg: „Gesellschaftliche Integration durch Binnenintegration?“, in: Kölner Zeitschrift für Soziologie und Sozialpsychologie (4) 1982, S. 717-731.

Endres, Jürgen et al.: Jung, muslimisch, schweizerisch. Muslimische Jugendgruppen, islamische Lebensführung und Schweizer Gesellschaft. Ein Forschungsbericht. Luzern: Universität Luzern, Zentrum Religionsforschung 2013.

Engelmann, Kerstin: Muslimische Weblogs. Der Islam im deutschsprachigen Internet (= Medien und politische Kommunikation - Naher Osten und islamische Welt 20). Berlin 2010.

Fürlinger, Ernst: Moscheebaukonflikte in Österreich. Nationale Politik des religiösen Raums im globalen Zeitalter. Wien 2015.

Gotzmann, Andreas et al.: Pluralismus in der Europäischen Religionsgeschichte. Religionswissenschaftliche Antrittsvorlesungen (= Europäische Religionsgeschichte 1). Marburg 2001.

Heine, Susanne / Lohlker, Rüdiger / Potz, Richard: Muslime in Österreich. Geschichte, Lebenswelt, Religion, Grundlagen für den Dialog. Innsbruck 2012.

Hüttermann, Jörg: Das Minarett. Zur politischen Kultur des Konflikts um islamische Symbole, Weinheim. München 2006.

Idriz, Benjamin: Grüß Gott, Herr Imam! Eine Religion ist angekommen. München 2010. Jasarevic, Alen: „Anders! Das Islamische Forum in Penzberg. Meine Erfahrungen als Architekt einer Moschee“, in: Beinhauer-Köhler, Bärbel / Leggewie, Claus: Moscheen in Deutschland. Religiöse Heimat und gesellschaftliche Herausforderung. München 2009, S. 99-111.

Kippenberg, Hans-G. / Rüpke, Jörg / von Stuckrad, Kocku (Hg.): Europäische Religionsgeschichte. Ein mehrfacher Pluralismus, Bd. 1. Göttingen 2009.

Klöcker, Michael / Tworuschka, Udo (Hg.): Praktische Religionswissenschaft - ein Handbuch für Studium und Beruf. Köln u. a. 2008.

Liebman, Robert C. / Sutton, John R. / Wuthnow, Robert: „Exploring the Social Sources of Denominationalism: Schisms in American Protestant Denominations, 1890-1980“, in: American Sociological Review (53/3) 1988, S. 343-352.

Müller, Monika: Migration und Religion. Junge hinduistische und muslimische Männer in der Schweiz. Wiesbaden 2013.

Nagel, Alexander-Kenneth (Hg.): Diesseits der Parallelgesellschaft. Neue Studien zu religiösen Migrantengemeinden in Deutschland. Bielefeld 2013. 
Nagel, Alexander-Kenneth (Hg.): Religiöse Netzwerke. Die zivilgesellschaftlichen Potentiale religiöser Migrantengemeinden. Bielefeld 2015.

Niebuhr, H. Richard: The Social Sources of Denominationalism. New York 1971 [1929].

Nowak, Jürgen: Leitkultur und Parallelgesellschaft. Argumente wider einen deutschen Mythos. Frankfurt am Main 2006.

Pahud de Mortanges, René (Hg.): Staatliche Anerkennung von Religionsgemeinschaften: Zukunfts- oder Auslaufmodell? Zürich / Genf 2015.

Polak, Regina / Reiss, Wolfram (Hg.): Religion im Wandel. Transformation religiöser Gemeinschaften in Europa durch Migration. Interdisziplinäre Perspektiven (= Religion and Transformation in Contemporary European Society 9). Wien 2015.

Reiss, Wolfram: „Auswirkungen der religiösen Pluralität auf staatliche Institutionen und die Anstaltsseelsorge“, in: Polak, Regina / Reiss, Wolfram (Hg.): Religion im Wandel. Transformation religiöser Gemeinschaften in Europa durch Migration. Interdisziplinäre Perspektiven (= Religion and Transformation in Contemporary European Society 9). Wien 2015, S. 147-186.

Reiss, Wolfram: „Zur Einleitung der Reihe ,Anwendungsorientierte Religionswissenschaft. Veränderungen der Schwerpunkte der Religionswissenschaft"“, in: Schönberger, Thomas: Der Islam im öffentlichen Bewusstsein. Marburg 2012, S. 5-25.

Scherer, Ross. P. (ed.): American Denominational Organisation. A Sociological View. Pasadena 1980.

Schlieter, Jens / Kind, Marietta / Lauer, Tina (Hg.): Die zweite Generation der Tibeter in der Schweiz: Identitätsaushandlungen und Formen buddhistischer Religion. Zürich 2014.

Schuller, Josef Peter: Die verborgene Moschee. Zur Sichtbarkeit muslimischer Gebetsräume in Wien (= Anwendungsorientierte Religionswissenschaft 4). Marburg 2013.

Stolz, Jörg et al.: Religion und Spiritualität in der Ich-Gesellschaft. Vier Gestalten des (Un-)Glaubens. Zürich 2014.

Stöckli, Lucia: Moschee-Neubauprojekte in England und der Schweiz. Institutionalisierung - Bedeutung - Sichtbarkeit (= Diss Universität Luzern). Luzern 2014.

Traunmüller, Richard: Religion als Ressource sozialen Zusammenhalts? Eine empirische Analyse der religiösen Grundlagen sozialen Kapitals in Deutschland (= SOEP Papers on Multidisciplinary Panel Data Research at DIW Berlin 144). Berlin 2008.

Traunmüller, Richard: „Religiöse Diversität und Sozialintegration im internationalen Vergleich“, in: Kölner Zeitschrift für Soziologie und Sozialpsychologie (65/1) 2013, S. 437-446.

Weiss, Karin / Thränhardt, Dietrich (Hg.): Selbsthilfe. Wie Migranten Netzwerke knüpfen und soziales Kapital schaffen. Freiburg im Breisgau 2005.

\section{Internetquellen}

Allievi, Stefano: Conflicts over Mosques in Europe. Policy Issues and Trends - NEF Initiative on Religion and Democracy in Europe, 2009, verfügbar unter: http://www. nef-europe.org/wp-content/uploads/2013/03/Conflicts-over-mosques_NEF-RelDemRELIGION-MOSQUES-Final-1.pdf [05.05.2016]. 
Alternative für Deutschland: Grundsatzprogramm der Alternative für Deutschland, Leitantrag der Bundesprogrammkommission und des Bundesvorstandes, Vorlage zum Bundesparteitag am 30.04.2016/01.05.2016, Stuttgart, verfügbar unter: https:// www.alternativefuer.de/wp-content/uploads/sites/7/2016/03/Leitantrag-Grundsatz programm-AfD.pdf [14.10.2016].

Antidiskriminierungsstelle des Bundes (Hg.): Akzeptanz religiöser und weltanschaulicher Vielfalt in Deutschland. Ergebnisse einer repräsentativen Umfrage im Auftrag der Antidiskriminierungsstelle des Bundes, Berlin 2016, verfügbar unter: http://www. antidiskriminierungsstelle.de/SharedDocs/Downloads/DE/publikationen/Umfragen/ Bericht_zur_Umfrage_Akzeptanz_religioeser_und_weltanschaulicher_Vielfalt_in_ Deutschland.pdf?_blob=publicationFile \&v=2 [27.09.2016].

Assheuer, Thomas: „Die neuen Feinde. Das Gerede von der ,christlich-jüdischen Leitkultur" schürt den Fremdenhass", in: DIE ZEIT, 23.10.2010, verfügbar unter: http:// www.zeit.de/2010/43/Leitkultur [02.01.2014].

Baier, Dirk et al.: Kinder und Jugendliche in Deutschland: Gewalterfahrungen, Integration, Medienkonsum. Zweiter Bericht zum gemeinsamen Forschungsprojekt des Bundesministeriums des Innern und des KFN (= Forschungsbericht Nr. 109), 2010, verfügbar unter: http://kfn.de/wp-content/uploads/Forschungsberichte/FB_109.pdf [04.05.2016].

Baumann, Martin / Tunger-Zanetti, Andreas: „Ansehen und Sichtbarmachung. Religion, Immigration und repräsentative Sakralbauten in Westeuropa“, in: Herder Korrespondenz (65/8) 2011, S. 407-409, verfügbar unter: https://www.unilu.ch/fileadmin/ shared/Publikationen/baumann-tunger_sichtbarkeit-ansehen_herder-korrespondenz -aug.2011.pdf [05.05.2016].

Bundesministerium für Bildung und Forschung: Islamische Theologie in Deutschland verankern, 19.01.2016, verfügbar unter: https://www.bmbf.de/de/islamische-theo logie-in-deutschland-verankern-2356.html [14.04.2016].

Bundesministerium für Inneres (Hg.): „Dialogforum Islam“, in: Magazin des Innenministeriums (1-2) 2013, verfügbar unter: http://www.bmi.gv.at/cms/BMI_Oeffentliche Sicherheit/2013/files/INTEGRATION_II.pdf [27.09.2016].

Church of Pentecost International Germany, Website, verfügbar unter: http://www.cop germany.com/ [14.04.2016].

Das Freiburger Bündnis: Argumente der Initiative gegen ein „Zentrum Islam und Gesellschaft“, verfügbar unter: http://www.udc-fr.ch/wp-content/uploads/Argumente_ D.pdf [17.09.2015].

Detjen, Stephan: „,Der Islam gehört zu Deutschland“. Die Geschichte eines Satzes“, in: Deutschlandfunk, 13.01.2015, verfügbar unter: http://www.deutschlandfunk.de/derislam-gehoert-zu-deutschland-die-geschichte-eines-satzes.1783.de.html?dram:article _id=308619 [18.09.2015].

Deutsche Islamkonferenz, Website, verfügbar unter: www.deutsche-islam-konferenz.de [28.09.2016].

Eidgenössischen Migrationskommission, Website, verfügbar unter: https://www.ekm. admin.ch/ekm/de/home.html [27.09.2016].

Focus Online: „Seehofer legt Sieben-Punkte-Plan nach“, in: Focus online, 16.10.2016, verfügbar unter: http://www.focus.de/politik/deutschland/integration-seehofer-legt-sie ben-punkte-plan-nach_aid_562723.html [14.10.2016]. 
Frings, Dorothea: Diskriminierung aufgrund der islamischen Religionszugehörigkeit im Kontext Arbeitsleben - Erkenntnisse, Fragen und Handlungsempfehlungen. Diskriminierungen von Musliminnen und Muslimen im Arbeitsleben und das AGG, Antidiskriminierungsstelle des Bundes (Hg.), Berlin 2010, verfügbar unter: http://www. antidiskriminierungsstelle.de/SharedDocs/Downloads/DE/publikationen/Experti sen/Expertise_Diskr_aufgrund_islam_Religionszugehoerigkeit_rechtswissenschaft lich.pdf?_blob $=$ publicationFile \& $v=1$ [06.05.2016].

Gleichbehandlungsanwaltschaft, Website, verfügbar unter: http://www.gleichbehand lungsanwaltschaft.at [27.09.2016].

Graduiertenkolleg „Kulturkontakt und Wissenschaftsdiskurs“ der Universität Rostock: Ziele des Graduiertenkollegs, verfügbar unter: http://www.gk-kulturkontakt.uni-ro stock.de/ziele/ [04.05.2016].

Hukuki.net: Türken, die kein Türkisch können, 24.09.2012, verfügbar unter: http://al manca.hukuki.net/turken-die-kein-turkisch-konnen.htm [14.04.2016].

Islamgesetz 2015. Bundesgesetz über die äußeren Rechtsverhältnisse islamischer Religionsgesellschaften, in: BGBl. I Nr. 39/2015, verfügbar unter: https://www.ris.bka.gv. at $/$ GeltendeFassung.wxe ? Abfrage $=$ Bundesnormen $\&$ Gesetzesnummer $=20009124$ [09.05.2016].

Jäggi, Sabine / Schär, Benz H.R.: „Gottes Volk hat viele Farben“. Migrationskirchen als Herausforderung und Chance für die Reformierten Kirchen Bern - Jura - Solothurn. Hg. Fachstelle Migration. Bern 2009, verfügbar unter: http://www.refbejuso.ch/file admin/user_upload/Downloads/OeME_Migration/Migration-Integration/OM_Pub_ Gottes_Volk_hat_viele_Farben.pdf [22.02.2016].

Jenkner, Carolin: „Moschee-Eröffnung. Warum das Wunder in Marxloh funktioniert“, in: Spiegel online, 26.10.2008, verfügbar unter: www.spiegel.de/politik/deutschland/ 0,1518,586613,00.html [05.08.2015].

Karimian, Shohreh: „Wir Türken haben immer noch ein Gastarbeiter-Image“, in: Qantara.de, 2014, verfügbar unter: http://de.qantara.de/inhalt/das-neue-gesicht-der-tages themen-pinar-atalay-wir-tuerken-haben-immer-noch-ein-gastarbeiter [14.04.2016].

Magistrat der Stadt Wien: Konzept der interkulturellen Projektarbeit der MA 17, verfügbar unter: https://www.wien.gv.at/menschen/integration/projektarbeit/konzept. html [09.05.2016].

Meier, Marcus: „Christlich-jüdische Leitkultur“? Fallstricke bei der Bildungsarbeit gegen Antisemitismus", in: Gesellschaftliche Zusammenhänge. Aus Politik und Zeitgeschichte (13-14), 18.3.2013, S. 57-62, verfügbar unter: http://www.bpb.de/apuz/ 156781/fallstrickebei-der-bildungsarbeit-gegen-antisemitismus [02.01.2014].

Moscheesuche, Website, verfügbar unter: http://www.moscheesuche.de/ [22.02.2016].

Peuker, Mario: Diskriminierung aufgrund der islamischen Religionszugehörigkeit im Kontext Arbeitsleben - Erkenntnisse, Fragen und Handlungsempfehlungen Erkenntnisse der sozialwissenschaftlichen Forschung und Handlungsempfehlungen, Hg. Antidiskriminierungsstelle des Bundes, Berlin 2010, verfügbar unter: http://www.an tidiskriminierungsstelle.de/SharedDocs/Downloads/DE/publikationen/Expertisen/ Expertise_Diskr_aufgrund_islam_Religionszugehoerigkeit_sozialwissenschaftlich. pdf?_blob $=$ publicationFile\&v $=1$ [06.05.2016].

Preuß, Ulrich K.: „Kein Ort, nirgends. Die vergebliche Suche nach der deutschen Leitkultur - Eine Replik auf Josef Isensee“, in: Blätter für die deutsche und internationale 
Politik (6) 2010, S. 67-79, verfügbar unter: http://www.blaetter.de/archiv/jahrgaenge/ 2010/juni/kein-ort-nirgends [02.01.2014].

Religionswissenschaftlicher Medien- und Informationsdienst e. V.: Religionen vor Ort Darstellungen und Untersuchungen, verfügbar unter: http://remid.de/projekte_ lokal_orte/ [27.09.2016].

Reiss, Wolfram: „Der Umgang mit religiösen Minderheiten in der österreichischen Armee“, in: Mattes, Astrid / Mourão Permoser, Julia / Stoeckl, Kristina (eds.): Institutional responses to religious diversity (= Interdisciplinary Journal for Religion and Transformation in Contemporary Society 2). Wien 2016, S. 82-113; DOI 10.14220/ .2016.02.issue-1, verfügbar unter: http://www.v-r.de/en/institutional_response_to_re ligious_diversity/c-3054 [09.05.2016].

Sachverständigenrat deutscher Stiftungen für Integration und Migration: Viele Götter, ein Staat: Religiöse Vielfalt und Teilhabe im Einwanderungsland. Jahresgutachten 2016 mit Integrationsbarometer, Berlin 2016, verfügbar unter: http://www.svr-migra tion.de/wp-content/uploads/2016/04/SVR_JG_2016-mit-Integrationsbarometer_ WEB.pdf [04.05.2016].

Stolz, Jörg / Chaves, Mark / Monnot, Christophe / Amiotte-Suchet, Laurent: Die religiösen Gemeinschaften in der Schweiz: Eigenschaften, Aktivitäten, Entwicklung. Schlussbericht der National Congregations Study Switzerland (NCSS) im Rahmen des Nationalen Forschungsprogramms 58, Lausanne 2011, verfügbar unter: http:// www.nfp58.ch/files/news/126_Schlussbericht_Stolz_Chaves.pdf [22.02.2016].

Stolz, Jörg / Könemann, Judith et al.: Religiosität in der modernen Welt. Bedingungen, Konstruktionen und sozialer Wandel, Schlussbericht im Nationalen Forschungsprogramm „Religion, Staat und Gesellschaft“, Lausanne 2011, verfügbar unter: http:// www.nfp58.ch/files/downloads/Schlussbericht_Stolz.pdf [22.02.2016].

Universität Freiburg: Schweizer Zentrum für Islam und Gesellschaft: Wissenschaftlicher Dialog ist notwendiger denn je, 28.01.2015, verfügbar unter: http://www.unifr.ch/ news/de/13745/ [17.09.2015].

Universität Luzern: Kuppel - Tempel - Minarett, verfügbar unter: www.unilu.ch/ktm [27.09.2016].

Wake Up International, Website, verfügbar unter: http://www.wkup.org/ [18.09.2015]. Wulff, Christian: „Vielfalt schätzen - Zusammenhalt fördern“. Rede zum 20. Jahrestag der Deutschen Einheit, 03.10.2010, verfügbar unter: http://www.bundespraesident. de/SharedDocs/Reden/DE/Christian-Wulff/Reden/2010/10/20101003_Rede.html [02.01.2014]. 